\title{
Prevalence of SARS-CoV-2 in household members and other close contacts of COVID-19 cases: a serologic study in canton of Vaud, Switzerland
}

Julien Dupraz ${ }^{a}$, Audrey Butty ${ }^{a}$, Olivier Duperrex ${ }^{a}$, Sandrine Estoppey ${ }^{a}$, Vincent Faivre ${ }^{a}$, Julien Thabard $^{a}$, Claire Zuppinger ${ }^{a}$, Gilbert Greub ${ }^{b, c}$, Giuseppe Pantaleo ${ }^{d, e}$, Jérôme Pasquier ${ }^{a}$, Valentin Rousson ${ }^{a}$, Malik Egger ${ }^{a}$, Amélie Steiner-Dubuis ${ }^{a}$, Sophie Vassaux ${ }^{a}$, Eric Masserey ${ }^{f}$, Murielle Bochud* $^{\text {a }}$, Semira Gonseth Nusslé* ${ }^{\text {a }}$, Valérie D’Acremont* a,g

* Contributed equally

a) Center for Primary Care and Public Health (Unisanté), University of Lausanne, Switzerland

b) Institute of Microbiology, Lausanne University Hospital and University of Lausanne, Switzerland

c) Service of Infectious Diseases, Lausanne University Hospital and University of Lausanne, Switzerland

d) Service of Immunology and Allergy, Lausanne University Hospital and University of Lausanne, Switzerland

e) Swiss Vaccine Research Institute, Lausanne University Hospital and University of Lausanne, Switzerland

f) Cantonal Medical Office, Department of Health and Social Action, Canton of Vaud, Switzerland

g) Swiss Tropical and Public Health Institute, University of Basel, Switzerland

\section{Addresses for correspondence}

1) Dr Julien Dupraz, Unisanté, route de la Corniche 10, 1010 Lausanne, Switzerland Julien.Dupraz@unisante.ch

Phone: +41 213147318

2) Prof. Valérie D'Acremont, Unisanté, route de Berne 113, 1010 Lausanne, Switzerland Valerie.DAcremont@unisante.ch

Phone: +41795562551

Key points: Risk of SARS-CoV-2 transmission within households is very high. Enforcing protective measures at home during COVID-19 episodes, as well as supporting self-isolation within the house by shortening the time between symptom onset and test result, could help mitigating such risk.

(C) The Author(s) 2021. Published by Oxford University Press on behalf of Infectious Diseases Society of America.

This is an Open Access article distributed under the terms of the Creative Commons AttributionNonCommercial-NoDerivs licence (http://creativecommons.org/licenses/by-nc-nd/4.0/), which permits non-commercial reproduction and distribution of the work, in any medium, provided the original work is not altered or transformed in any way, and that the work is properly cited. For commercial re-use, please contact journals.permissions@oup.com 


\section{Abstract}

\section{Background}

Research on SARS-CoV-2 transmission within households and other close settings using serological testing is scarce.

\section{Methods}

We invited COVID-19 cases diagnosed between February 27 and April 1, 2020, in canton of Vaud, Switzerland, to participate, along with household members and other close contacts. Anti-SARS-CoV2 IgG antibodies were measured using a Luminex immunoassay. We estimated factors associated with serological status using generalized estimating equations.

\section{Results}

Overall, 219 cases, 302 household members, and 69 other close contacts participated between May 4 and June 27,2020 . More than half of household members $(57.2 \%, 95 \% \mathrm{Cl} 49.7-64.3)$ had developed a serologic response to SARS-CoV-2, while $19.0 \%(95 \% \mathrm{Cl} 10.0-33.2)$ of other close contacts were seropositive. After adjusting for individual and household characteristics, infection risk was higher in household members aged 65 or more than in younger adults (aOR $3.63,95 \% \mathrm{Cl} 1.05-12.60)$, and in those not strictly adhering to simple hygiene rules like hand washing (aOR $1.80,95 \% \mathrm{Cl} 1.02-3.17)$. The risk was lower when more than 5 people outside home were met during semi-confinement, compared to none $(\mathrm{aOR} 0.35,95 \% \mathrm{Cl} 0.16-0.74)$. Individual risk of household members to be seropositive was lower in large households (22\% less per each additional person). 


\section{Conclusions}

During semi-confinement, household members of a COVID-19 case were at very high risk of getting infected, 3 times more than close contacts outside home. This highlights the need to provide clear messages on protective measures applicable at home. For elderly couples, who were especially at risk, providing external support for daily basic activities is essential.

Keywords: SARS-CoV-2; transmission; household; serology; COVID-19 


\section{Introduction}

The understanding of transmission patterns is especially critical to guide interventions aiming at limiting the occurrence of new cases of coronavirus disease 2019 (COVID-19). In this respect, transmission of severe acute respiratory syndrome coronavirus 2 (SARS-CoV-2) in promiscuous settings such as households is of particular interest and is at the core of the early investigation protocols provided by the World Health Organization (WHO Unity Studies) to address the many unknowns related to the COVID-19 pandemic ${ }^{1,2}$.

Studies dealing with the transmission of SARS-CoV-2 within households have found secondary attack rates (SAR) ranging from 3.9 to $44.6 \%$, reflecting heterogeneous settings and study designs ${ }^{3}$. The evidence regarding transmission to close contacts outside the household tends to show lower SAR (from 0.7 to $5.1 \%$ ), but attack rates above $50 \%$ have been reported in certain circumstances ${ }^{4-8}$. Most studies conducted so far are based on the identification of active disease through nucleic acid amplification tests (NAAT), whose sensitivity can be hampered by various factors ${ }^{9}$.

The availability of serological assays allows the identification of past infection and thus provides key input into our understanding of the epidemiology of SARS-CoV-2. Nevertheless, studies on SARSCoV-2 transmission in close settings using serological testing remain scarce. So far, most of them found SAR close to $35 \%$ within households ${ }^{10-13}$. However, none of them includes a thorough investigation of factors associated with seropositivity. Regarding close contacts outside the household, research shows SAR ranging from 0 to $13.7 \%$, but study designs and settings are

disparate ${ }^{12,14-16}$. Furthermore, the amount of available serological assays is quickly growing, often with limited external validation of their accuracy, and concerns are emerging regarding their accuracy in the setting of seroepidemiological studies because of the lower median level of antibodies in participants compared to clinical studies ${ }^{17}$. 
This work was part of SerocoViD, a community-based seroepidemiological study of SARS-CoV-2 infection conducted in canton of Vaud, Switzerland, embedded within a nationwide program, Corona Immunitas ${ }^{18}$. Taking advantage of prior development and validation of a highly sensitive serological assay carried out locally ${ }^{19}$, the objective was to determine the prevalence of anti-SARSCoV-2 IgG antibodies among household members and other close contacts of COVID-19 cases, and to identify factors associated with seropositivity in these highly exposed people.

\section{Methods}

\section{Study design and participants}

SerocoViD is a cross-sectional community-based seroepidemiological study of SARS-CoV-2 infection conducted in canton of Vaud (French-speaking region of Switzerland, 806'088 inhabitants on December 31, 2019). The study was launched at the end of April 2020, coinciding with the easing of semi-confinement measures taken in Switzerland in mid-March. From February 27 (first confirmed case in canton of Vaud) to March 4, 2020, all COVID-19 cases underwent contact tracing by local authorities. At that time, a close contact was any individual who had been within two meters of an infected person for at least 15 minutes, starting 24 hours before illness onset. Given the exponential growth of the number of cases, contact tracing was stopped from week 2 of the epidemic. For the same reason, from March 9, 2020, diagnostic testing was limited to healthcare personal, hospitalized people and individuals at increased risk for severe illness in the entire country.

We sampled confirmed COVID-19 cases from the cantonal registry (total $n \approx 3^{\prime} 700$ ). With the exception of three people (one deceased, two who returned home abroad), all confirmed cases from week 1 were invited to participate in the study $(n=13)$, along with their close contacts identified by contact tracing $(n=117)$. Additionally, all cases aged between 6 months and 19 years $(n=66)$ and a random sample of non-institutionalized cases aged 20 and more $(n=368)$ who were tested positive 
during weeks 2 to 5 (from March 5 to April 1, 2020) were invited to take part in the study. In order to extend the age range of confirmed cases for whom a contact tracing procedure had been performed, the study team conducted complementary tracing procedures for three adolescent cases, thus identifying 20 additional close contacts outside the household.

Overall, this resulted in the solicitation of 447 confirmed cases (called thereafter index cases) and 137 close contacts not belonging to the household of index cases. Moreover, index case participants were asked to invite all their household members aged 6 months or more to take part in the study. Because of testing restrictions, index cases were not necessarily the first infected in their household, but those fulfilling testing criteria. All index cases were diagnosed using NAAT.

\section{Patient consent statement}

The Cantonal Ethics Committee of Vaud, Switzerland, approved the protocol (ID 2020-00887), and written consent was obtained from participants.

\section{Procedures}

Index cases and their close contacts identified by contact tracing were invited by letters. Participants registered into the study and answered the study questionnaire (available in French and English) via an online platform. The questionnaire covered the following topics: socio-demographic information, medical history, history of symptoms compatible with COVID-19 and use of health services, living conditions and household characteristics, contacts with other people in private and professional settings, and compliance with measures aimed at controlling the epidemic. The full questionnaire is available as supplementary material.

Study visits took place in four centers distributed over the cantonal territory between May 4 and June 27, 2020. A venous blood sample was collected to proceed with serological testing. We offered a home visit by a mobile study team to people at increased risk for severe illness from COVID-19. All participants (or their legal representative) provided written informed consent. 


\section{Detection of anti-SARS-CoV-2 antibodies}

We measured anti-SARS-CoV-2 IgG antibodies targeting the spike (S) protein in its native trimeric form using a Luminex immunoassay. This test was developed by the Lausanne University Hospital, Switzerland, in collaboration with the École Polytechnique Fédérale de Lausanne (EPFL), and compared with five commercially available immunoassays detecting IgG against the $\mathrm{N}$ protein and the monomeric moieties of the S1 protein ${ }^{19}$. The in-house Luminex S protein trimer IgG assay was 99.2\% specific in sera from people infected with pre-pandemic coronaviruses or from patients with autoimmune diseases, and proved to be more sensitive $(96.7 \%)$ than commercial tests in hospitalized patients with moderate to severe disease 16 to 33 days post-symptoms. The threshold for a positive result was defined at an antibody Multiplex Fluorescent Immunoassay (MFI) ratio of $\geq 6$.

\section{Statistical analysis}

We calculated the proportion of index cases with a positive serology test result and computed a Clopper-Pearson $95 \%$ confidence interval $(95 \% \mathrm{Cl})$. Significant clustering of infections within households has been reported in previous research ${ }^{20}$. In order to account for correlation between close contacts of a same index case, we used generalized estimating equations (GEE) with an exchangeable correlation structure to estimate the seroprevalence and corresponding $95 \% \mathrm{Cl}$ among contacts. Odds ratios (OR) were computed to measure the strength of the association between each independent variable and serology test result. We used GEE to account for correlation between contacts of a same index case and calculated OR with their $95 \% \mathrm{Cl}$ and $\mathrm{p}$-value using a logit link function. Finally, a multivariable regression model using GEE was fitted to measure the adjusted association of individual and household characteristics with serology test result among household members. Considering the potential influence of past diagnostic testing for SARS-CoV-2 on the reporting of symptoms, we proceeded to a sensitivity analysis among contacts not reporting 
previous nasal or throat swabbing. We performed statistical analysis using Stata/IC version 16.1. There was no imputation of missing values.

\section{Results}

Two-hundred and nineteen index cases (49.0\%), aged 2 to 90 years (mean 48.7, SD 19.3), participated in the study, of which $55.7 \%$ considered themselves as women. They reported 421 household members, of which 302 (71.7\%), aged 1 to 87 years (mean 37.0, SD 21.3), took part in the study. Sixty-nine (50.4\%) close contacts outside the household, aged 9 to 85 years (mean 47.8 , SD 17.0), participated.

\section{Prevalence of seropositivity in the different groups}

Most index cases $(215 / 219,98.2 \%)$ had a positive serological test result (95\% Cl 95.4-99.5; figure 1). The crude proportion of positives was $53.0 \%$ in household members (160/302) and $17.4 \%$ among close contacts outside the household (12/69). When taking into account correlation, the seroprevalence was $57.2 \%$ in household members $(95 \% \mathrm{Cl} 49.7-64.3)$ and $19.0 \%$ in close contacts outside the household $(95 \% \mathrm{Cl} 10.0-33.2)$.

\section{Unadjusted association of individual and household characteristics with seropositivity (bivariable} analysis)

A higher proportion of household members aged 65 to $75(85.7 \%)$ and 75 or more (83.3\%) were seropositive (table 1). No association between serological test result and gender or level of education was found (figure 2). Household members currently smoking had lower odds of infection than non-smokers in bivariable analysis (unadjusted $\mathrm{OR} 0.56,95 \% \mathrm{Cl} 0.32-0.96$ ). In close contacts outside the household, seroprevalence was $30.3 \%$ and $5.7 \%$ in overweight/obese and normal/underweight participants, respectively, but no association was found in household members. Close contacts not strictly adhering to simple hygiene rules tended to have higher odds of 
infection (table 2, figure 2). We found no association between serology and the compliance with social distancing rules. Positive test results were less frequent in household members who had met more than five people per week during the semi-confinement compared to none (unadjusted OR $0.42,95 \% \mathrm{Cl} 0.22-0.78)$, but there was no association with the number of close encounters with symptomatic individuals. In bivariable analysis, seroprevalence significantly decreased with increasing household size. We found that $66.1 \%$ of participants living with one other person only (the index COVID-19 case) had a positive test result, contrasting with participants living with five people or more, who showed a $26.0 \%$ risk to be seropositive (unadjusted OR $0.19,95 \% \mathrm{Cl} 0.06-0.62$ ). There was an inverse relationship between household size and mean age of participants in the household (supplementary table 1).

Adjusted association of individual and household characteristics with seropositivity (multivariable analysis)

We finally estimated the adjusted association of individual and household characteristics with serology test result among household members (table $\mathbf{3}$ ). The odds of infection were almost four times higher in household members aged 65 or more than in the younger age group (adjusted OR $3.63,95 \% \mathrm{Cl} 1.05-12.60)$. The association of current smoking with negative serology observed in bivariable analysis faded in multivariable model (adjusted $\mathrm{OR} 0.73,95 \% \mathrm{Cl} 0.38-1.39$ ). Although overweight/obesity tended to be associated with higher odds of infection, this association was not statistically significant at the 0.05 level. In comparison with bivariable analysis, we observed a strengthening of the relation between the absence of strict adherence to simple hygiene rules and positive serology testing (adjusted $\mathrm{OR} 1.80,95 \% \mathrm{Cl} 1.02-3.17$ ). However, there was no indication of a link with adherence to social distancing rules or mask wearing. The association of a greater number of social contacts during the semi-confinement with lower odds of infection was confirmed in multivariable analysis (adjusted $\mathrm{OR} 0.35,95 \% \mathrm{Cl} 0.16-0.74$ ). On the other hand, close encounters with symptomatic individuals tended to be associated with positive serology, but this tendency was not 
statistically significant at the 0.05 level. Household characteristics did not show a significant association with serological test result. Adding characteristics of the index case to the model (age, gender) yielded comparable estimates (results not shown).

\section{Prevalence and clinical presentation of flu-like episodes, and use of health services}

The occurrence of one flu-like episode or more since the end of February 2020 was strongly associated with positive serological testing, both in household members (OR 3.55, 95\% Cl 2.37-5.32, table 4) and close contacts outside the household (OR 8.64, 95\% $1.77-42.12$ ). The proportion of asymptomatic seropositive individuals (i.e. not reporting any flu-like episode) was $21.4 \%$ in household members, and $16.7 \%$ in close contacts outside the household. With the exception of chest pain, all reported symptoms were associated with a positive serology. This was particularly evident in household members mentioning new-onset anosmia or ageusia, of which $92.8 \%$ were seropositive (OR 6.24, 95\% $\mathrm{Cl} 3.46-11.24$ ). When limiting the analysis to participants not reporting previous nasal or throat swabbing, the strength of the association between symptoms and serology generally increased (supplementary table 2). Half the seropositive household members not mentioning prior PCR testing reported tiredness (49.6\%; figure 3 ), followed by headache (44.1\%), cough $(37.1 \%)$, fever $(36.8 \%)$, aching muscle or joints $(36.6 \%)$, and anosmia or ageusia $(35.9 \%)$. Gastrointestinal symptoms were infrequent. Half of seropositive household members (46.3\%) reported contact with a medical provider, and $6.3 \%$ were hospitalized. Figures were comparable among seropositive close contacts outside the household (41.7\% and $8.3 \%$, respectively). However, hospitalization rate was higher in index cases (14.7\%).

\section{Discussion}

More than one in two participants living with a confirmed COVID-19 case has developed a serologic response to SARS-CoV-2, while one in five close contacts outside the household was seropositive. Our findings confirm that households represent high-risk transmission settings $4,5,8,12,21-23$. The SAR 
we observed is substantially higher than the one reported in previous seroepidemiological studies, including a large nationwide survey conducted in Spain (37.4\%), and a retrospective cohort study conducted in Singapore (11\%, estimation based on Bayesian modelling $)^{11-13,23}$. One study disclosed a SAR of $80 \%$ in household members of essential workers, but estimation was based on 30 participants only ${ }^{10}$. Beside serological testing characteristics, differences could be due to variable average household sizes (2.2 members in Switzerland vs 2.6 in Spain) ${ }^{24}$, unequal adoption of protective behaviors within households ${ }^{25}$, or different levels of confinement. Regarding close contacts outside the household, previous seroepidemiological studies provided SAR estimations ranging from 0 to $13.7 \%^{12,14-16,23}$. Heterogeneity of results could reflect different study designs and settings, and varying adherence to public health protective recommendations ${ }^{25}$. The strong difference observed between the prevalence in household members and in close contacts outside home is probably due the fact that contacts at home are closer and last longer than outside, due to the difficulty of applying social distancing in limited spaces and with family members. Moreover, simple hygiene rules may be more neglected at home, maybe due to a feeling of security.

We found that older household members were at particularly high risk, corroborating the findings of previous research on transmission using NAAT ${ }^{8,26,27}$. This association was not found for close contacts outside home. This suggests that elderly couples are even less able to apply protective measures at home, due to their high level of mutual dependency. There was no difference in infection susceptibility according to gender, which is in line with other works ${ }^{5,23,26}$. The impact of smoking on the risk of SARS-CoV-2 infection is a controversial issue ${ }^{28}$. Although household members currently smoking were less frequently positive, this association vanished in multivariable analysis, suggesting that it may be confounded by other factors. The importance of hygiene measures to avoid transmission within household is confirmed by our observations ${ }^{29}$. Mask wearing in public and respect of social distancing rules, which is particularly difficult when living under the same roof, were not associated with infection risk in households. In contrast, the association of a greater number of social contacts with a lower probability of infection seems surprising at first place. In fact, our study 
took place during a period of semi-confinement, during which most people stayed at home, except those who had to go out to work in essential sectors. Our findings thus show that the individual risk of being infected is higher when staying at home than working outside, the aim of confinement (or quarantine) being to break the transmission chain. We have thus to accept that this works well but at the price of a higher risk for household members of COVID-19 cases to be infected. Like previous studies, we found an inverse relationship between household size and the proportion of seropositive household members ${ }^{26,27}$. This seems counter-intuitive, as prevalence of infectious diseases is well known to be associated with crowded housing. However, being many in a household allows decreasing mutual dependency and thus close contacts. This association was weakened by inclusion of the mean age of household members in the multivariable model, suggesting that the apparent protective effect of a high number of household members could reflect the fact that large families are, on average younger. However, disentangling respective contributions of household size and age distribution of household members remains difficult.

Regarding the clinical presentation of COVID-19, the proportion of asymptomatic seropositive individuals was close to findings of Pollán and colleagues in Spain (28.5\%) ${ }^{12}$. Even if not specific, a large number of symptoms were still associated with SARS-CoV-2 infection, especially new-onset smell and/or taste disturbance, confirming the clinical utility of this symptom to suspect COVID-19 ${ }^{30}$. Interestingly, the prevalence of flu-like symptoms was high also in seronegative people, maybe because the first epidemic wave occurred just after the winter, when other respiratory infections were still quite prevalent.

Limitations need to be acknowledged. The Swiss testing policy during the first epidemic wave, which limited diagnostic testing mainly to individuals at increased risk for severe illness, made the sample of index cases not representative of all cases that occurred in the community during this period. Index cases were thus not necessarily the first infected in their household, but those fulfilling testing 
criteria. However, this would be especially problematic if the purpose were to identify factors associated with infectivity of the index case, which we deliberately avoided.

Incidence of new COVID-19 cases remains high worldwide and prevention of transmission is, for now, the only way to tackle the pandemic. If concerns regarding the transmission of SARS-CoV-2 in shops, restaurants and public gatherings are justified, our findings emphasize that the risk of being infected is much higher at home. However, this remains overlooked in collective awareness and public health discourse, precisely because quarantine and confinement are methods used to break the transmission chain. Early testing of the first case in a household is important to support immediate self-isolation within the house. Our results suggest in particular that it is essential for non-institutionalized elderly couples to receive strong external support for daily basic needs during the infectious period of the index case. Further research is needed to determine the efficacy and acceptability of specific measures aimed at limiting SARS-CoV-2 transmission within households and at motivating early testing and self-isolation. 


\section{Author contributions}

JD did the statistical analyses and drafted the first version of the manuscript. JD, MB, SGN and VDA designed the article, accessed the data and contributed to the interpretation of data. MB, SGN and VDA conceived and conducted the study, and contributed to drafting sections of the manuscript. JD, $A B, O D, S E, V F, J T, C Z, M E, A S D, S V, M B, S G N$ and VDA participated in planning of the study and collection of data. GG and GP were involved in development and validation of the serological test. JP and VR provided support for planning and performing statistical analyses. EM contributed to study design. All authors commented on drafts, read and approved the final manuscript. The corresponding author attests that all listed authors meet authorship criteria and that no others meeting the criteria have been omitted.

\section{Funding}

This work was supported by the operating budget of the Center for Primary Care and Public Health (Unisanté), University of Lausanne, Switzerland, and by contributions of local health authorities (Department of Health and Social Action, Canton of Vaud) and the following Swiss non-profit institutions: Leenaards Foundation, Fondation pour l'Université de Lausanne. SerocoViD is part of the national Corona Immunitas program coordinated by the Swiss School of Public Health Plus $(\mathrm{SSPH}+)$.

\section{Potential conflicts of Interest}

The authors declare no competing interests. 


\section{Data sharing}

All data and materials used in this work are accessible to researchers upon reasonable request for data sharing to the corresponding author.

\section{Acknowledgments}

The authors would like to thank warmly all study participants for their involvement. This study was made possible by the strong involvement of all the SerocoViD operational team (Julia Baldwin, Giovanna Bonsembiante-Poidomani, Ophélie Hoffmann, Emilie Jendly, Athiththan Kanthasami, Daria Mapelli, Virginie Schlüter, Kevin Schutzbach, Auriane Soris and Lucie Wuillemin). 


\section{References}

1. Pitzer VE, Cohen T. Household studies provide key insights on the transmission of, and susceptibility to, SARS-CoV-2. Lancet Infect Dis. Published online June 17, 2020. doi:10.1016/S14733099(20)30514-4

2. Unity Studies: Early Investigation Protocols. Accessed June 30, 2020.

https://www.who.int/emergencies/diseases/novel-coronavirus-2019/technical-guidance/earlyinvestigations

3. Madewell ZJ, Yang Y, Longini IM, Halloran ME, Dean NE. Household transmission of SARSCoV-2: a systematic review and meta-analysis of secondary attack rate. medRxiv. Published online August 1, 2020:2020.07.29.20164590. doi:10.1101/2020.07.29.20164590

4. Park YJ, Choe YJ, Park O, et al. Contact Tracing during Coronavirus Disease Outbreak, South Korea, 2020. Emerg Infect Dis. 2020;26(10). doi:10.3201/eid2610.201315

5. Wu Y, Song S, Kao Q, Kong Q, Sun Z, Wang B. Risk of SARS-CoV-2 infection among contacts of individuals with COVID-19 in Hangzhou, China. Public Health. 2020;185:57-59. doi:10.1016/j.puhe.2020.05.016

6. Hamner L. High SARS-CoV-2 Attack Rate Following Exposure at a Choir Practice - Skagit County, Washington, March 2020. MMWR Morb Mortal Wkly Rep. 2020;69. doi:10.15585/mmwr.mm6919e6

7. Böhmer MM, Buchholz U, Corman VM, et al. Investigation of a COVID-19 outbreak in Germany resulting from a single travel-associated primary case: a case series. Lancet Infect Dis. Published online May 15, 2020. doi:10.1016/S1473-3099(20)30314-5 
8. Cheng H-Y, Jian S-W, Liu D-P, et al. Contact Tracing Assessment of COVID-19 Transmission Dynamics in Taiwan and Risk at Different Exposure Periods Before and After Symptom Onset. JAMA Intern Med. Published online May 1, 2020. doi:10.1001/jamainternmed.2020.2020

9. Caruana G, Croxatto A, Coste AT, et al. Diagnostic strategies for SARS-CoV-2 infection and interpretation of microbiological results. Clin Microbiol Infect. Published online June 25, 2020. doi:10.1016/j.cmi.2020.06.019

10. McDade TW, McNally EM, Zelikovich AS, et al. High seroprevalence for SARS-CoV-2 among household members of essential workers detected using a dried blood spot assay. PloS One. 2020;15(8):e0237833. doi:10.1371/journal.pone.0237833

11. Silveira MF, Barros AJD, Horta BL, et al. Population-based surveys of antibodies against SARSCoV-2 in Southern Brazil. Nat Med. Published online July 8, 2020. doi:10.1038/s41591-020-0992-3

12. Pollán M, Pérez-Gómez B, Pastor-Barriuso R, et al. Prevalence of SARS-CoV-2 in Spain (ENECOVID): a nationwide, population-based seroepidemiological study. The Lancet. 2020;396(10250):535-544. doi:10.1016/S0140-6736(20)31483-5

13. Cox RJ, Brokstad KA, Krammer F, Langeland N, Bergen COVID-19 Research Group. Seroconversion in household members of COVID-19 outpatients. Lancet Infect Dis. Published online June 15, 2020. doi:10.1016/S1473-3099(20)30466-7

14. Brown NE, Bryant-Genevier J, Bandy U, et al. Antibody Responses after Classroom Exposure to Teacher with Coronavirus Disease, March 2020. Emerg Infect Dis. 2020;26(9). doi:10.3201/eid2609.201802

15. Zhang H-J, Su Y-Y, Xu S-L, et al. Asymptomatic and symptomatic SARS-CoV-2 infections in close contacts of COVID-19 patients: a seroepidemiological study. Clin Infect Dis. Published online June 16, 2020. doi:10.1093/cid/ciaa771 
16. Chu VT, Freeman-Ponder B, Lindquist S, et al. Investigation and Serologic Follow-Up of Contacts of Early Confirmed Case-Patient with COVID-19, United States. Emerg Infect Dis. 2020;26(8). doi:10.3201/eid2608.201423

17. Deeks JJ, Dinnes J, Takwoingi Y, et al. Antibody tests for identification of current and past infection with SARS-CoV-2. Cochrane Database Syst Rev. 2020;6:CD013652. doi:10.1002/14651858.CD013652

18. West EA, Anker D, Amati R, et al. Corona Immunitas: study protocol of a nationwide program of SARS-CoV-2 seroprevalence and seroepidemiologic studies in Switzerland. Int J Public Health. Published online October 24, 2020. doi:10.1007/s00038-020-01494-0

19. Fenwick C, Croxatto A, Coste AT, et al. Changes in SARS-CoV-2 Spike versus Nucleoprotein Antibody Responses Impact the Estimates of Infections in Population-Based Seroprevalence Studies. J Virol. Published online November 3, 2020. doi:10.1128/JVI.01828-20

20. Stringhini S, Wisniak A, Piumatti G, et al. Seroprevalence of anti-SARS-CoV-2 IgG antibodies in Geneva, Switzerland (SEROCoV-POP): a population-based study. The Lancet. 2020;0(0). doi:10.1016/S0140-6736(20)31304-0

21. Bi Q, Wu Y, Mei S, et al. Epidemiology and transmission of COVID-19 in 391 cases and 1286 of their close contacts in Shenzhen, China: a retrospective cohort study. Lancet Infect Dis. Published online April 27, 2020. doi:10.1016/S1473-3099(20)30287-5

22. Gudbjartsson DF, Norddahl GL, Melsted P, et al. Humoral Immune Response to SARS-CoV-2 in Iceland. N Eng/ J Med. Published online September 1, 2020. doi:10.1056/NEJMoa2026116

23. Ng OT, Marimuthu K, Koh V, et al. SARS-CoV-2 seroprevalence and transmission risk factors among high-risk close contacts: a retrospective cohort study. Lancet Infect Dis. Published online November 2, 2020. doi:10.1016/S1473-3099(20)30833-1 
24. United Nations, Department of Economic and Social Affairs, Population Division. Database on Household Size and Composition 2019. Accessed August 27, 2020.

https://population.un.org/Household/index.html

25. Clark C, Davila A, Regis M, Kraus S. Predictors of COVID-19 voluntary compliance behaviors: An international investigation. Glob Transit. 2020;2:76-82. doi:10.1016/j.glt.2020.06.003

26. Jing Q-L, Liu M-J, Zhang Z-B, et al. Household secondary attack rate of COVID-19 and associated determinants in Guangzhou, China: a retrospective cohort study. Lancet Infect Dis. 2020;0(0). doi:10.1016/S1473-3099(20)30471-0

27. Rosenberg ES, Dufort EM, Blog DS, et al. COVID-19 Testing, Epidemic Features, Hospital Outcomes, and Household Prevalence, New York State-March 2020. Clin Infect Dis. Published online May 8, 2020. doi:10.1093/cid/ciaa549

28. Usman MS, Siddiqi TJ, Khan MS, et al. Is there a smoker's paradox in COVID-19? BMJ EvidBased Med. Published online August 11, 2020. doi:10.1136/bmjebm-2020-111492

29. Wu J, Huang Y, Tu C, et al. Household Transmission of SARS-CoV-2, Zhuhai, China, 2020. Clin Infect Dis. Published online May 11, 2020. doi:10.1093/cid/ciaa557

30. Makaronidis J, Mok J, Balogun N, et al. Seroprevalence of SARS-CoV-2 antibodies in people with an acute loss in their sense of smell and/or taste in a community-based population in London, UK: An observational cohort study. PLoS Med. 2020;17(10):e1003358.

doi:10.1371/journal.pmed.1003358 
Table 1: Serology test result according to general characteristics and medical history, stratified by type of participant (unadjusted results)

\begin{tabular}{|c|c|c|c|c|c|c|c|}
\hline & $\begin{array}{c}\text { Index cases } \\
(\mathbf{N}=\mathbf{2 1 9}) \\
\mathrm{n}(\%) \text { seropositive }\end{array}$ & $\mathrm{n}(\%)$ seropositive & $\begin{array}{l}\text { d members }(\mathrm{N}=\mathbf{3 0 2}) \\
\text { Unadjusted OR } \\
{[95 \% \mathrm{Cl}]}\end{array}$ & $\mathrm{p}$-value & $\mathrm{n}(\%)$ seropositive & $\begin{array}{l}\text { ide the household } \\
\text { Unadjusted OR } \\
{[95 \% \mathrm{Cl}]}\end{array}$ & $\mathrm{V}=69$ ) \\
\hline All participants & 215/219 (98.2) & $160 / 302(53.0)$ & & & $12 / 69(17.4)$ & & \\
\hline Age & & & & 0.119 & & & 0.928 \\
\hline $6 m o-<5 y$ & $1 / 2(50.0)$ & $5 / 11(45.5)$ & 0.92 [0.37-2.29] & & no participant & .. & \\
\hline $5 y-<10 y$ & $1 / 1(100.0)$ & $12 / 22(54.6)$ & $1.16[0.55-2.44]$ & & $0 / 1(0.0)$ & .. & \\
\hline $10 y-<15 y$ & $2 / 2(100.0)$ & $15 / 32$ (46.9) & 0.93 [0.49-1.75] & & $0 / 3(0.0)$ & .. & \\
\hline $15 y-<20 y$ & $20 / 21(95.2)$ & $9 / 19(47.4)$ & $1.17[0.56-2.45]$ & & $0 / 2(0.0)$ & .. & \\
\hline $20 y-<40 y$ & 42/43 (97.7) & $37 / 76$ (48.7) & reference & & $3 / 15(20.0)$ & reference & \\
\hline $40 y-<65 y$ & 103/104 (99.0) & $60 / 116$ (51.7) & $0.83[0.52-1.30]$ & & $7 / 38(18.4)$ & $0.92[0.22-3.87]$ & \\
\hline $65 y-<75 y$ & $31 / 31(100.0)$ & $12 / 14$ (85.7) & $3.98[1.03-15.44]$ & & $2 / 9(22.2)$ & $1.32[0.16-11.00]$ & \\
\hline $75 y$ or more & $15 / 15(100.0)$ & $10 / 12(83.3)$ & $5.25[1.16-23.72]$ & & $0 / 1(0.0)$ & $\cdot$ & \\
\hline Gender & & & & 0.164 & & & 0.124 \\
\hline male & $94 / 96$ (97.9) & 74/146 (50.7) & reference & & $8 / 30(26.7)$ & reference & \\
\hline female & $120 / 122(98.4)$ & $86 / 156$ (55.1) & 1.27 [0.91-1.76] & & 4/39 (10.3) & 0.36 [0.10-1.32] & \\
\hline other & $1 / 1(100.0)$ & no participant &.$\cdot$ & & no participant &.$\cdot$ & \\
\hline Current smoker ${ }^{1}$ & & & & 0.034 & & & 0.825 \\
\hline no & 191/194 (98.5) & $142 / 256(55.5)$ & reference & & 10/59 (17.0) & reference & \\
\hline yes & 23/24 (95.8) & 18/46 (39.1) & $0.56[0.32-0.96]$ & & 2/9 (22.2) & $1.21[0.22-6.53]$ & \\
\hline Weight status & & & & 0.146 & & & 0.011 \\
\hline normal or underweight & 104/106 (98.1) & $92 / 184(50.0)$ & reference & & $2 / 35(5.7)$ & reference & \\
\hline $\begin{array}{l}\text { overweight or obese } \\
\text { Adult participants only }\end{array}$ & $110 / 112(98.2)$ & $66 / 111(59.5)$ & $1.37[0.90-2.09]$ & & $10 / 33(30.3)$ & $6.74[1.54-29.50]$ & \\
\hline Education & & & & 0.196 & & & 0.423 \\
\hline lower secondary or less & $21 / 21(100.0)$ & $21 / 33(63.6)$ & reference & & $3 / 10(30.0)$ & reference & \\
\hline upper secondary & $58 / 59(98.3)$ & $46 / 82(56.1)$ & $0.93[0.46-1.90]$ & & $5 / 23(21.7)$ & $0.64[0.13-3.21]$ & \\
\hline tertiary & $108 / 109(99.1)$ & $51 / 100(51.0)$ & $0.61[0.30-1.24]$ & & $4 / 30(13.3)$ & $0.33[0.06-1.83]$ & \\
\hline Chronic medical conditions $^{2}$ & & & & 0.037 & & & 0.148 \\
\hline none & $131 / 133(98.5)$ & $86 / 169$ (50.9) & reference & & $6 / 44(13.6)$ & reference & \\
\hline one or more & $58 / 58(100.0)$ & $32 / 47(68.1)$ & $1.94[1.04-3.62]$ & & $6 / 19(31.6)$ & $2.48[0.73-8.48]$ & \\
\hline Hypertension & & & & 0.110 & & & 0.034 \\
\hline no & 152/154 (98.7) & $95 / 182(52.2)$ & reference & & $7 / 50(14.0)$ & reference & \\
\hline yes & $36 / 36(100.0)$ & 21/31 (67.7) & $1.81[0.87-3.74]$ & & $5 / 11(45.5)$ & $4.48[1.12-18.01]$ & \\
\hline Diabetes & & & & 0.489 & & & 0.082 \\
\hline no & 169/171 (98.8) & $111 / 206(53.9)$ & & & $10 / 57(17.5)$ & reference & \\
\hline yes & $15 / 15(100.0)$ & $4 / 6(66.7)$ & $1.75[0.36-8.48]$ & & $2 / 3(66.7)$ & $8.59[0.76-96.92]$ & \\
\hline Cardiovascular disease & & & & 0.239 & & & .. \\
\hline no & 170/172 (98.8) & $103 / 196(52.6)$ & reference & & $12 / 58(20.7)$ & .. & \\
\hline yes & $12 / 12(100.0)$ & $9 / 12(75.0)$ & $2.03[0.62-6.62]$ & & $0 / 2(0.0)$ & .. & \\
\hline Kidney disease & & & & .. & & & .. \\
\hline no & 181/183 (98.9) & $115 / 212(54.3)$ & .. & & $12 / 60(20.0)$ & .. & \\
\hline yes & $3 / 3(100.0)$ & no participant &.$\cdot$ & & $0 / 1(0.0)$ & .. & \\
\hline Chronic respiratory disease & & & & 0.196 & & & .. \\
\hline no & $177 / 179(98.9)$ & $106 / 202(52.5)$ & reference & & $12 / 59(20.3)$ & .. & \\
\hline yes & $7 / 7(100.0)$ & $4 / 5(80.0)$ & $3.79[0.50-28.52]$ & & $0 / 2(0.0)$ & .. & \\
\hline Immunodeficiency & & & & 0.596 & & & .. \\
\hline no & $174 / 176(98.9)$ & $110 / 202(54.5)$ & reference & & $12 / 58(20.7)$ & .. & \\
\hline yes & $12 / 12(100.0)$ & 4/9 (44.4) & $0.74[0.25-2.23]$ & & $0 / 3(0.0)$ & .. & \\
\hline Cancer & & & & .. & & & .. \\
\hline no & $177 / 179(98.9)$ & $113 / 209(54.1)$ & .. & & $12 / 58(20.7)$ & .. & \\
\hline yes & 4/4 (100.0) & no participant &.. & & $0 / 2(0.0)$ & .. & \\
\hline Other chronic condition & & & & 0.325 & & & .. \\
\hline & 159/160 (99.4) & $97 / 184(52.7)$ & reference & & $12 / 55(21.8)$ & .. & \\
\hline yes & $24 / 25(96.0)$ & $18 / 28(64.3)$ & $1.49[0.68-3.27]$ & & $0 / 7(0.0)$ & .. & \\
\hline
\end{tabular}

Calculation of odds ratio and p-value: correlation between close contacts of a same index case taken into account using GEE (exchangeable correlation structure, logit link function). ${ }^{1}$ Children aged less than 12 considered non-smokers. ${ }^{2}$ Among all following conditions, except "other chronic condition". 
Table 2: Serology test result according to adherence to measures aimed at decreasing transmission, contacts with other people, and living conditions, stratified by type of participant (unadjusted results)

\begin{tabular}{|c|c|c|}
\hline & $\begin{array}{r}\text { Househo } \\
\mathrm{n}(\%) \text { seropositive }\end{array}$ & $\begin{array}{c}\text { nembers } \mathbf{( N = 3 0 2} \\
\text { Unadjusted OR } \\
{[95 \% \mathrm{Cl}]}\end{array}$ \\
\hline \multicolumn{3}{|l|}{ Respect of measures and contacts with other people } \\
\hline \multicolumn{3}{|l|}{$\begin{array}{l}\text { Respect of simple hygiene rules (washing hands regularly, sneezing } \\
\text { into the elbow, etc.) }\end{array}$} \\
\hline $\begin{array}{lll}2 y e s & \text { yes }\end{array}$ & $114 / 215(53.0)$ & referenc \\
\hline rather yes & $40 / 74(54.1)$ & $1.46[0.97-2.2 c$ \\
\hline rather no or no & $4 / 9(44.4)$ & $2.08[0.63-6.8]$ \\
\hline
\end{tabular}

Respect of social distancing rules (physical distancing, avoid shaking hands or kissing, etc.)

Wearing a mask in public

$$
\begin{array}{r}
\text { yes } \\
\text { rather yes } \\
\text { rather no or no }
\end{array}
$$$$
\text { no }
$$$$
\begin{array}{r}
\text { yes, sometimes } \\
\text { yes, always }
\end{array}
$$

Weekly number of people met outside home during the semiconfinement

none
1 to 5
more than 5
Close contact with people outside home having symptoms Close contact with people
suggestive of COVID-19

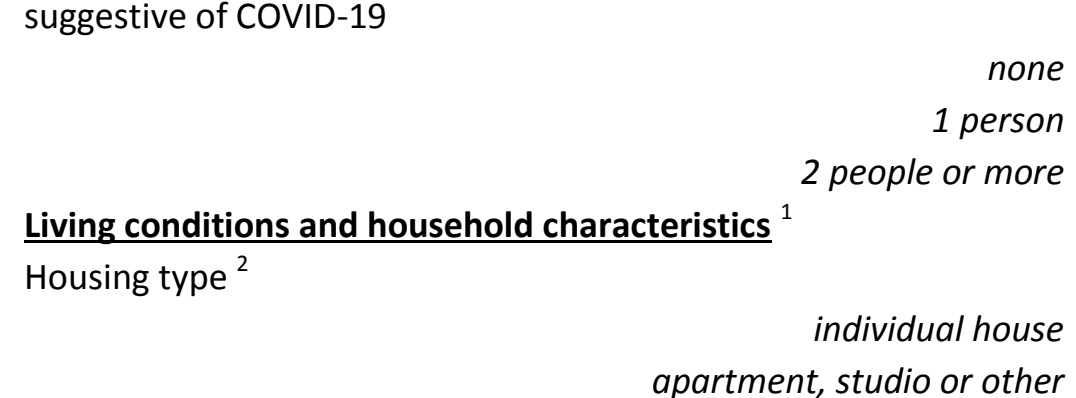

Number of people in the household ${ }^{2}$

Number of habitable rooms (besides kitchen) in the dwelling ${ }^{3}$

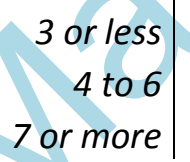$$
87 / 157(55.4)
$$$$
56 / 105(53.3)
$$$$
15 / 36(41.7)
$$

$$
\begin{array}{r}
79 / 163(48.5) \\
54 / 98(55.1) \\
27 / 40(67.5)
\end{array}
$$

$$
\begin{array}{r}
\text { reference } \\
0.99[0.64-1.52] \\
0.77[0.42-1.43]
\end{array}
$$

(0.7)

$1.15[0.75-1.74]$

$1.31[0.70-2.45]$

$65 / 100(65.0)$

21/61 (34.4)

$127 / 233$ (54.5)

$17 / 34(50.0)$

16/35 (45.7)

reference

0.87 [0.53-1.43]

0.42 [0.22-0.78]

$$
\text { (2) }
$$

reference

1.11 [0.63-1.96]

$83 / 158(52.5)$

$77 / 144(53.5)$

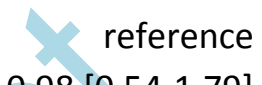

0.98 [0.54-1.79]

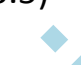

$39 / 59$ (66.1)

$31 / 51(60.8)$

44/74 (59.5)

$33 / 68$ (48.5)

$13 / 50(26.0)$

$35 / 54(64.8)$

$96 / 183(52.5)$

29/65 (44.6)

reference
$0.82[0.35-1.93]$
$0.78[0.34-1.77]$
$0.43[0.17-1.06]$
$0.19[0.06-0.62]$

reference

0.67 [0.32-1.40]

0.55 [0.21-1.47]
$73 / 140(52.1)$

$1.48[0.80-2.75]$$$
2.08 \text { [0.63-6.87] }
$$

reference

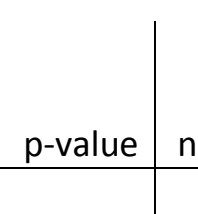

Close contacts outside the household ( $N=69$ ) Unadjusted OR $[95 \% \mathrm{Cl}]$ p-value

\subsection{3}

\%) seropositive

$(95 \% \mathrm{Cl}$

pent

$\begin{array}{rr}8 / 56(14.3) & \text { reference } \\ 4 / 13(30.8) & 2.22[0.54-9.21] \\ \text { no participant } & . .\end{array}$

0.114

$7 / 53$ (13.2) reference

$5 / 15(33.3) \quad 2.94$ [0.77-11.23]

$0 / 1(0.0)$

0.093

$5 / 38(13.2) \quad$ reference

2/17 (11.8) $\quad 1.06[0.21-5.35]$

$5 / 13(38.5) \quad 4.36[1.06-17.83]$

0.761

2/17 (11.8) reference

6/34 (17.7) $\quad 1.26[0.25-6.35]$

$4 / 17(23.5) \quad 1.86[0.32-10.75]$

0.456

2/14 (14.3)

$5 / 12(41.7) \quad 4.36[0.68-27.99]$

0.099

All participants, including children and teens. Calculation of odds ratio and p-value: correlation between close contacts of a same index case taken into account using GEE (exchangeable correlation structure, logit link function). ${ }^{1}$ Not relevant for close contacts outside the household. ${ }^{2}$ Answer of the index case taken for all household members. ${ }^{3}$ Answer of the index case taken for all household members, except two households where information from index case was missing (mean of answers reported by other household members taken instead). 


\section{Characteristics of household member}

Age (ref: $20 y-<65 y$ )

Gender (ref: male)

Current smoker ${ }^{1}$ (ref: no)

Weight status (ref: normal or underweight)

Respect of simple hygiene rules (washing hands regularly, sneezing into the elbow, etc.) (ref: yes)

Respect of social distancing rules (physical distancing, avoid shaking hands or kissing, etc.) (ref: yes)

Wearing a mask in public (ref: no)

$1.02[0.61-1.72]$

Weekly number of people met outside home during the semi-confinement (ref: 0)

Close contact with people outside home having symptoms suggestive of COVID-19 (ref: none)

\section{Characteristics of household}

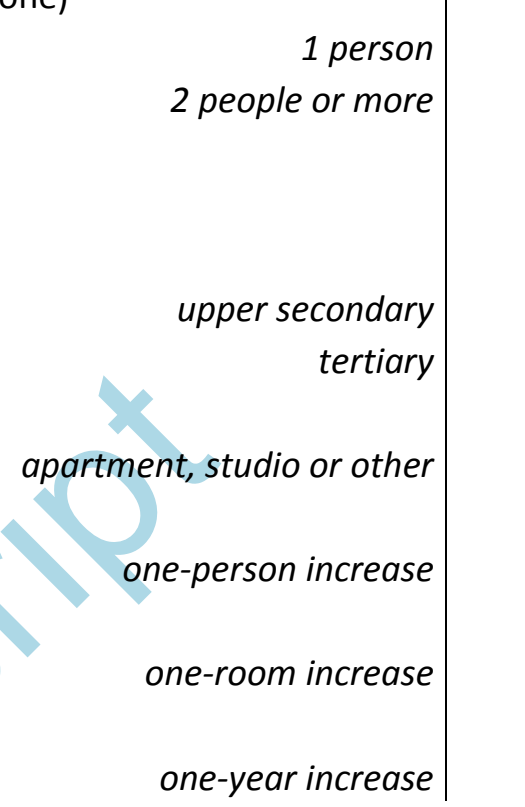

$0.70[0.40-1.21]$

Highest education level among adult household members (ref: lower secondary or less)

Housing type ${ }^{2}$ (ref: individual house)

Number of people in the household ${ }^{2}$

Number of habitable rooms (besides kitchen) in the dwelling ${ }^{3}$

Mean age of participating household members ${ }^{4}$

Multivariable regression model; 291/302 household members included in model. Within-household correlation taken into account using GEE (exchangeable correlation structure, logit link function). The variable "chronic medical conditions", that was not available for children and teens, was not included in the model. ${ }^{1}$ Children aged less than 12 considered non-smokers. ${ }^{2}$ Answer of the index case taken for all household members. ${ }^{3}$ Answer of the index case taken for all household members, except two households where information from index case was missing (mean of answers reported by other household members taken instead). ${ }^{4}$ Given the association between household size and mean age of participating household members, this variable was included in the model. 


\begin{tabular}{|c|c|c|c|c|c|c|c|}
\hline & $\begin{array}{c}\text { Index cases } \\
(\mathrm{N}=\mathbf{2 1 9})\end{array}$ & Househol & d members ( $N=302)$ & & Close contacts ou & tside the household (I & $N=69)$ \\
\hline & $\mathrm{n}(\%)$ seropositive & $\mathrm{n}(\%)$ seropositive & Odds ratio $[95 \% \mathrm{Cl}]$ & $\mathrm{p}$-value & $\mathrm{n}(\%)$ seropositive & Odds ratio $[95 \% \mathrm{Cl}]$ & p-value \\
\hline Symptoms & & & & & & & \\
\hline Flu-like episodes & & & & 0.000 & & & 0.008 \\
\hline none & $25 / 26(96.2)$ & $34 / 117(29.1)$ & reference & & $2 / 39(5.1)$ & reference & \\
\hline one or more & 190/193 (98.5) & $125 / 184(67.9)$ & $3.55[2.37-5.32]$ & & $10 / 29(34.5)$ & $8.64[1.77-42.12]$ & \\
\hline Cough & & & & 0.001 & & & 0.003 \\
\hline no & $58 / 58(100.0)$ & $84 / 192(43.8)$ & reference & & $5 / 53(9.4)$ & reference & \\
\hline yes & $157 / 161(97.5)$ & $76 / 110(69.1)$ & $2.07[1.36-3.15]$ & & $7 / 16(43.8)$ & $7.76[2.02-29.84]$ & \\
\hline Runny or stuffy nose, sneezing & & & & 0.001 & & & 0.008 \\
\hline no & 105/107 (98.1) & $93 / 208(44.7)$ & reference & & $5 / 52(9.6)$ & reference & \\
\hline yes & $110 / 112(98.2)$ & $67 / 94(71.3)$ & $2.18[1.38-3.44]$ & & $7 / 17(41.2)$ & $6.33[1.64-24.46]$ & \\
\hline Sore throat & & & & 0.049 & & & 0.729 \\
\hline no & $137 / 140(97.9)$ & $109 / 224(48.7)$ & reference & & $8 / 52(15.4)$ & reference & \\
\hline yes & 78/79 (98.7) & $51 / 78(65.4)$ & $1.53[1.00-2.33]$ & & $4 / 17(23.5)$ & $1.27[0.33-4.84]$ & \\
\hline Dyspnea & & & & 0.000 & & & 0.203 \\
\hline no & $123 / 125(98.4)$ & $108 / 235(46.0)$ & reference & & $9 / 61(14.8)$ & reference & \\
\hline yes & 92/94 (97.9) & $52 / 67(77.6)$ & $2.86[1.74-4.70]$ & & $3 / 8(37.5)$ & $2.82[0.57-13.97]$ & \\
\hline Feeling of fever & & & & 0.000 & & & 0.901 \\
\hline no & 93/95 (97.9) & $87 / 206(42.2)$ & reference & & 9/54 (16.7) & reference & \\
\hline yes & $122 / 124(98.4)$ & 73/96 (76.0) & $2.74[1.71-4.40]$ & & $3 / 15(20.0)$ & $1.09[0.27-4.44]$ & \\
\hline Temperature $37.5^{\circ} \mathrm{C}$ or more (measured) & & & & 0.000 & & & 0.007 \\
\hline no & $80 / 82(97.6)$ & $82 / 209(39.2)$ & reference & & $8 / 63(12.7)$ & reference & \\
\hline yes & $135 / 137(98.5)$ & 78/93 (83.9) & $4.64[2.82-7.65]$ & & 4/6 (66.7) & $12.61[1.98-80.32]$ & \\
\hline Headache & & & & 0.000 & & & 0.001 \\
\hline no & 79/81 (97.5) & $76 / 180(42.2)$ & reference & & $4 / 52(7.7)$ & reference & \\
\hline yes & $136 / 138(98.6)$ & $84 / 122(68.9)$ & 2.14 [1.43-3.19] & & $8 / 17(47.1)$ & $10.29[2.55-41.62]$ & \\
\hline Pain in muscles, joints & & & & 0.000 & & & 0.940 \\
\hline no & $86 / 89(96.6)$ & $88 / 203(43.4)$ & reference & & $10 / 58(17.2)$ & reference & \\
\hline yes & 129/130 (99.2) & 72/99 (72.7) & 2.44 [1.61-3.70] & & 2/11 (18.2) & $0.94[0.18-4.84]$ & \\
\hline Chest pain & & & & 0.444 & & & 0.018 \\
\hline no & $150 / 152(98.7)$ & $131 / 258(50.8)$ & reference & & $7 / 58(12.1)$ & reference & \\
\hline yes & 65/67 (97.0) & 29/44 (65.9) & $1.23[0.72-2.09]$ & & $5 / 11(45.5)$ & 5.56 [1.34-23.07] & \\
\hline Tiredness, exhaustion & & & & 0.000 & & & 0.001 \\
\hline no & $31 / 33$ (93.9) & $62 / 162(38.3)$ & reference & & $3 / 49(6.1)$ & reference & \\
\hline yes & $184 / 186(98.9)$ & $98 / 140(70.0)$ & 2.66 [1.79-3.95] & & $9 / 20(45.0)$ & $11.03[2.64-45.97]$ & \\
\hline Appetite loss & & & & 0.000 & & & 0.003 \\
\hline no & 89/93 (95.7) & $104 / 237(43.9)$ & reference & & $7 / 61(11.5)$ & reference & \\
\hline yes & $126 / 126(100.0)$ & $56 / 65(86.2)$ & $4.52[2.50-8.17]$ & & $5 / 8(62.5)$ & $11.30[2.28-55.89]$ & \\
\hline Nausea, vomiting & & & & 0.004 & & & 0.181 \\
\hline no & $178 / 182(97.8)$ & $138 / 275(50.2)$ & reference & & $11 / 67(16.4)$ & reference & \\
\hline yes & $37 / 37(100.0)$ & $22 / 27(81.5)$ & $2.93[1.40-6.13]$ & & $1 / 2(50.0)$ & $6.51[0.42-101.56]$ & \\
\hline Diarrhea & & & & 0.001 & & & 0.475 \\
\hline no & $154 / 157(98.1)$ & $123 / 250(49.2)$ & reference & & $10 / 62(16.1)$ & reference & \\
\hline yes & $61 / 62(98.4)$ & $37 / 52(71.2)$ & 2.37 [1.41-3.99] & & $2 / 7(28.6)$ & $1.90[0.33-11.00]$ & \\
\hline Belly pain & & & & 0.002 & & & 0.530 \\
\hline no & $175 / 178(98.3)$ & $134 / 267(50.2)$ & reference & & $10 / 62(16.1)$ & reference & \\
\hline yes & $40 / 41(97.6)$ & $26 / 35(74.3)$ & $2.76[1.44-5.28]$ & & $2 / 7(28.6)$ & $1.75[0.31-10.01]$ & \\
\hline Sudden loss of smell or taste & & & & 0.000 & & & 0.001 \\
\hline & 74/77 (96.1) & $96 / 233(41.2)$ & reference & & $6 / 62(9.7)$ & reference & \\
\hline & $141 / 142(99.3)$ & $64 / 69(92.8)$ & $6.24[3.46-11.24]$ & & $6 / 7$ (85.7) & $65.25[5.47-779.10]$ & \\
\hline
\end{tabular}




\begin{tabular}{|c|c|c|c|c|c|c|c|}
\hline \multirow[t]{2}{*}{ Table 4: continued } & \multirow{2}{*}{$\begin{array}{c}\text { Index cases } \\
(\mathbf{N = 2 1 9 )} \\
\mathrm{n}(\%) \text { seropositive }\end{array}$} & \multicolumn{3}{|c|}{ Household members $(\mathrm{N}=302)$} & \multicolumn{3}{|c|}{ Close contacts outside the household ( $N=69$ ) } \\
\hline & & $\mathrm{n}(\%)$ seropositive & Odds ratio $[95 \% \mathrm{Cl}]$ & p-value & $\mathrm{n}(\%)$ seropositive & Odds ratio $[95 \% \mathrm{Cl}]$ & p-value \\
\hline \multicolumn{8}{|l|}{ Use of health services } \\
\hline Contact with a medical provider & & & & 0.000 & & & 0.027 \\
\hline no & $28 / 28(100.0)$ & $86 / 209(41.2)$ & reference & & $7 / 57(12.3)$ & reference & \\
\hline yes & 185/189 (97.9) & 74/93 (79.6) & $3.61[2.19-5.95]$ & & $5 / 12(41.7)$ & $4.62[1.20-17.86]$ & \\
\hline Nasal or throat swabbing to detect SARS-CoV-2 & & & & 0.000 & & & 0.002 \\
\hline no &.$\cdot\left({ }^{1}\right)$ & $110 / 240(45.8)$ & reference & & $7 / 63(11.1)$ & reference & \\
\hline yes & $\cdot \cdot\left({ }^{1}\right)$ & $50 / 62(80.7)$ & $2.74[1.61-4.65]$ & & $5 / 6(83.3)$ & $38.52[3.95-375.53]$ & \\
\hline \multicolumn{8}{|l|}{ Test result for SARS-CoV-2 ${ }^{2}$} \\
\hline negative or unknown & $\cdot \cdot\left({ }^{1}\right)$ & $5 / 16(31.3)$ & .. & & $4 / 5(80.0)$ & .. & \\
\hline positive & $\cdot \cdot\left({ }^{1}\right)$ & $45 / 46(97.8)$ & .. & & $1 / 1(100.0)$ & .. & \\
\hline Hospitalization & & & & 0.028 & & & \\
\hline no & $181 / 185(97.8)$ & $150 / 291(51.6)$ & reference & & $11 / 68(16.2)$ & .. & \\
\hline yes & $32 / 32(100.0)$ & $10 / 11$ (90.9) & 4.96 [1.19-20.62] & & $1 / 1(100.0)$ & .. & \\
\hline \multicolumn{8}{|l|}{ Admission to the ICU } \\
\hline no & 203/207 (98.1) & $158 / 300(52.7)$ & .. & & $12 / 69(17.4)$ & .. & \\
\hline yes & $10 / 10(100.0)$ & $2 / 2(100.0)$ &.. & & no participant & .. & \\
\hline \multicolumn{8}{|l|}{ Intubation } \\
\hline no & $210 / 214(98.1)$ & $160 / 302(53.0)$ &.$\cdot$ & & $12 / 69(17.4)$ & .. & \\
\hline yes & $3 / 3(100.0)$ & no participant & .. & & no participant & .. & \\
\hline
\end{tabular}

All participants, including children and teens. Calculation of odds ratio and p-value: correlation between close contacts of a same index case taken into account using GEE (exchangeable correlation structure, logit link function). ${ }^{1}$ Index cases all tested positive. ${ }^{2}$ Nucleic acid amplification test. 
Figure 1: Percentage of participants with a positive serology test result, by type of participant

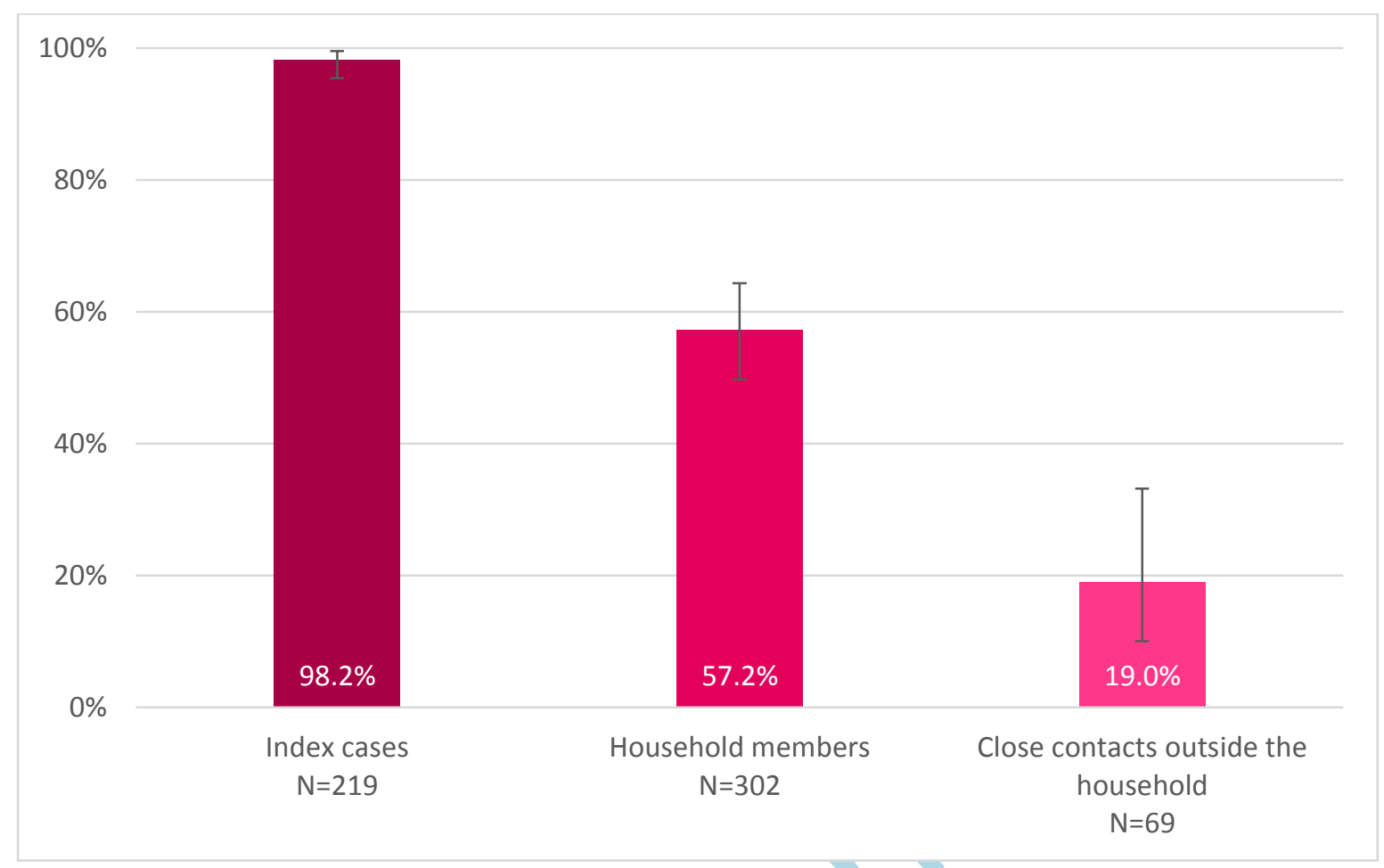

Index cases: crude proportion, calculation of $95 \%$ confidence interval using the Clopper-Pearson method. Household members and close contacts outside the household: proportion and corresponding 95\% confidence interval estimated using GEE (exchangeable correlation structure). 
Figure 2: Unadjusted association between characteristics of household members of index cases and seropositivity (bivariable analysis)

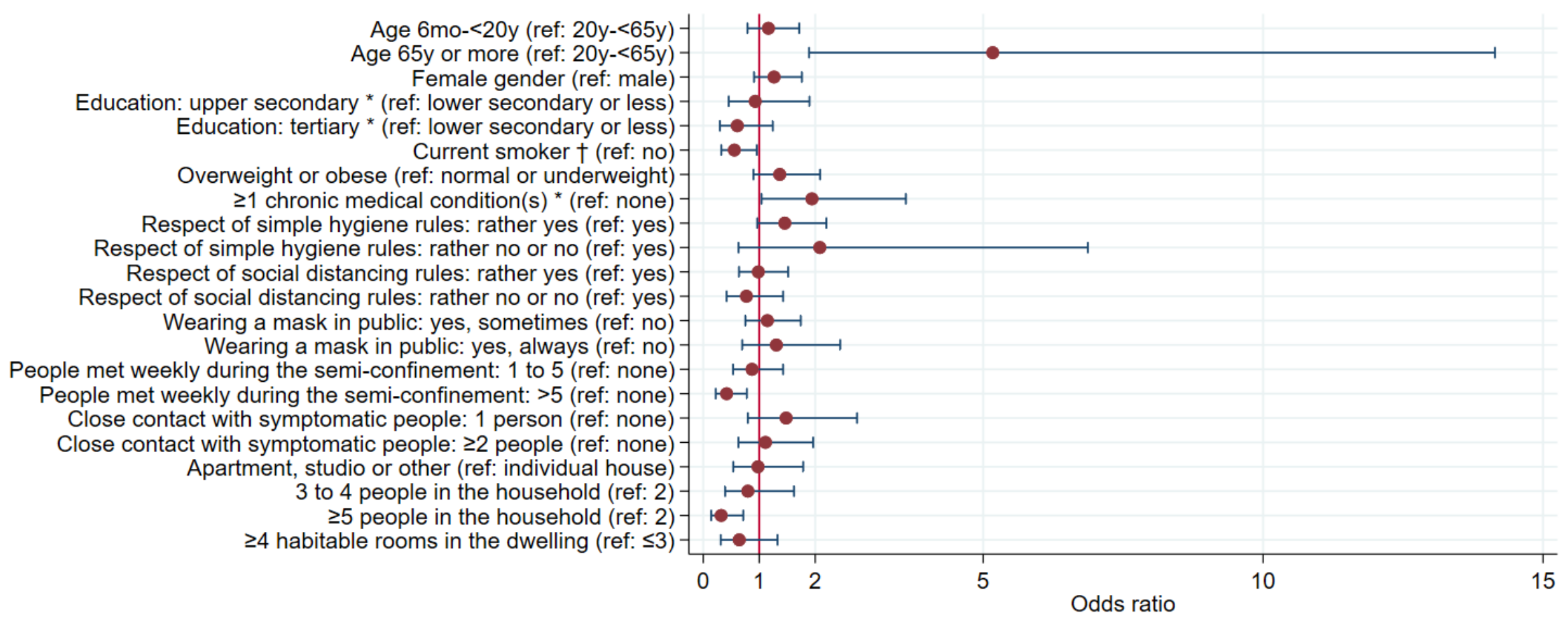

Calculation of odds ratio: correlation between household members of a same index case taken into account using GEE (exchangeable correlation structure,

logit link function). Error bars represent the limits of the $95 \%$ confidence interval for the odds ratio. * Adult participants only. + Children aged less than 12

considered non-smokers. 
Figure 3: Percentage of household members reporting specific symptoms, according to serology test result

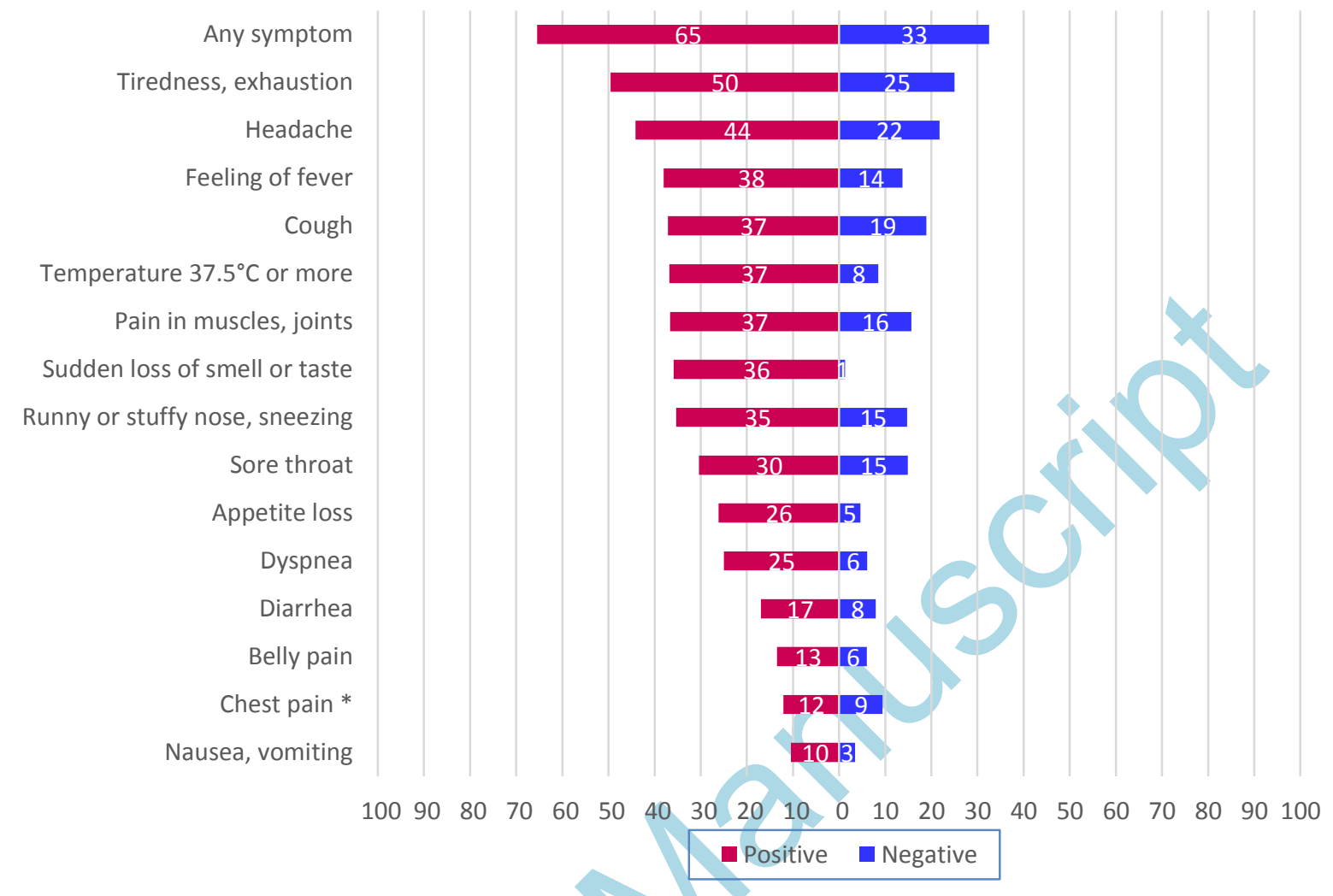

Household members not reporting prior nasal or throat swabbing to detect SARS-CoV-2. Correlation between household members of a same index case taken into account using GEE (exchangeable correlation structure). ${ }^{*}$ Difference not statistically significant at the 0.05 level. 